\title{
Chemisorption of Anionic Species from the Electrolyte Alters the Surface Electronic Structure and Composition of Photocharged $\mathrm{BiVO}_{4}$
}

\author{
Nienke J. Firet, ${ }^{\dagger, \perp}$ Anirudh Venugopal, ${ }^{\dagger, \perp}$ Marijn A. Blommaert, ${ }^{\dagger}$ C Chiara Cavallari,

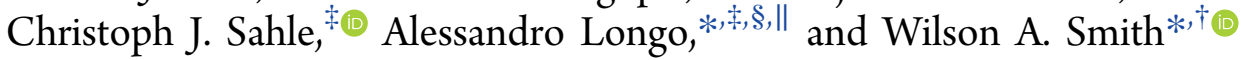 \\ ${ }^{\dagger}$ Materials for Energy Conversion and Storage (MECS), Department of Chemical Engineering, Delft University of Technology, Van \\ der Maasweg 9, 2629 HZ Delft, The Netherlands \\ "European Synchrotron Radiation Facility, The European Synchrotron, 71 Avenue des Martyrs, 38000 Grenoble, France \\ ${ }^{\S}$ Department of Materials, Textiles and Chemical Engineering, Ghent University, Technologiepark 125, 9052 Ghent, Belgium \\ "Istituto per lo Studio dei Materiali Nanostrutturati (ISMN)-CNR, UOS Palermo, Via Ugo La Malfa, 153, 90146 Palermo, Italy
}

Supporting Information

\begin{abstract}
Photocharging has recently been demonstrated as a powerful method to improve the photoelectrochemical water splitting performance of different metal oxide photoanodes, including $\mathrm{BiVO}_{4}$. In this work, we use ambient-pressure $\mathrm{X}$-ray Raman scattering (XRS) spectroscopy to study the surface electronic structure of photocharged $\mathrm{BiVO}_{4}$. The $\mathrm{O} \mathrm{K}$ edge spectrum was simulated using the finite difference method near-edge structure program package, which revealed a change in electron confinement and occupancy in the conduction band. These insights, combined with ultraviolet-visible spectroscopy and X-ray photoelectron spectroscopy analyses, reveal that a surface layer formed during photocharging creates a heterojunction with $\mathrm{BiVO}_{4}$, leading to

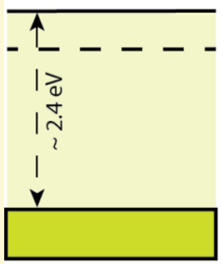

Reference BivO4

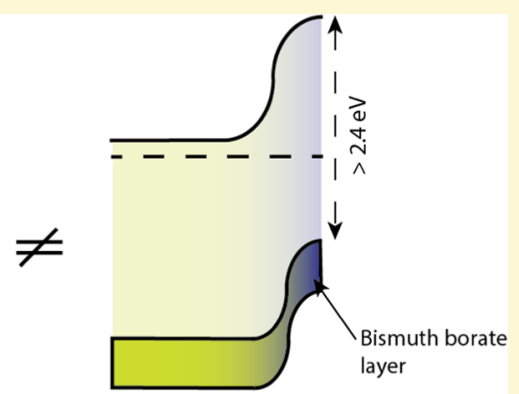

Photocharged BiVO4 favorable band bending and strongly reduced surface recombination. The XRS images presented in this work exhibit good agreement with soft X-ray absorption near-edge structure spectra from the literature, demonstrating that XRS is a powerful tool to study the electronic and structural properties of light elements in semiconductors. Our findings provide direct evidence of the electronic modification of a metal oxide photoanode surface as a result of the adsorption of electrolyte anionic species under operating conditions. This work highlights that the surface adsorption of these electrolyte anionic species is likely present in most studies on metal oxide photoanodes and has serious implications for the photoelectrochemical performance analysis and fundamental understanding of these materials.
\end{abstract}

\section{INTRODUCTION}

Bismuth vanadate $\left(\mathrm{BiVO}_{4}\right)$ is an extensively investigated $\mathrm{n}$ type metal oxide semiconductor that has shown promising properties for photoelectrochemical (PEC) water splitting. ${ }^{1}$ It has generated the highest photocurrent for a metal oxide photoanode to date, ${ }^{2}$ and the band gap of $2.4 \mathrm{eV}$ could potentially be suitable to make $\mathrm{BiVO}_{4}$ a good top absorber in tandem PEC devices with a low band gap bottom absorber. ${ }^{3}$ In addition, the optoelectronic properties of $\mathrm{BiVO}_{4}$ are easily modified: the material can accommodate a large number of defects and dopants, enabling a high degree of material engineering making $\mathrm{BiVO}_{4}$ a suitable model photoanode. ${ }^{4}$ Many improvements in the PEC performance of $\mathrm{BiVO}_{4}$ have used the addition of dopants to improve electron conductivity 3,5 or the addition of co-catalysts to improve the kinetics of the oxygen evolution reaction (OER).,

Interestingly, recent work by Zachäus et al. ${ }^{8}$ showed that surface recombination is one of the major performance- limiting factors for $\mathrm{BiVO}_{4}$. In fact, the presence of a cobalt phosphate $(\mathrm{CoPi})$ catalyst on the surface of $\mathrm{BiVO}_{4}$ suppresses surface recombination by aiding the transfer of holes from the semiconductor valence band (VB) to the OER intermediates. While CoPi effectively suppresses surface recombination, it also introduces parasitic light absorption, which is unwanted in a practical device. ${ }^{9}$ Recently, the photocharging (PC) technique has been introduced on $\mathrm{BiVO}_{4}$ photoanodes, which drastically reduced the onset potential for OER and increased the photocurrent density and fill factor of $\mathrm{PC}-\mathrm{BiVO}_{4}$ compared to untreated $\mathrm{BiVO}_{4} \cdot{ }^{10}$ This treatment was performed by exposing $\mathrm{BiVO}_{4}$ photoanodes to prolonged exposure of AM1.5 illumination under open-circuit conditions in a neutral electrolyte. After extensive material character-

Received: May 30, 2019

Revised: July 19, 2019

Published: July 19, 2019 
ization, it was shown that the surface structure of $\mathrm{BiVO}_{4}$ was altered upon photocharging. ${ }^{10,11}$ This treatment lead to a suppression of the surface recombination of photogenerated charge carriers, improving the overall photoelectrochemical performance of $\mathrm{BiVO}_{4}$. In a follow-up work, the $\mathrm{pH}$ of the electrolyte was found to have a significant effect on the photocharging enhancement, where an alkaline solution increased the PEC performance more compared to a neutral or slightly acidic electrolyte. ${ }^{11}$ Using intensity-modulated photoelectron spectroscopy and a hole scavenger, Liu et al. quantified the enhancement from photocharging in the bulk and at the surface of $\mathrm{BiVO}_{4}$ in a potassium phosphate buffer. ${ }^{12}$ It was demonstrated that photocharging of $\mathrm{BiVO}_{4}$ resulted in a significant enhancement in both the bulk charge separation and surface charge transfer efficiencies. Similar photoelectrochemical enhancements have also been recently shown in other metal oxide photoanodes such as $\mathrm{WO}_{3},{ }^{13} \mathrm{CuWO}_{4},{ }^{14}$ and doped $\mathrm{Fe}_{2} \mathrm{O}_{3},{ }^{15,16}$ showing that the photocharging treatment can be extended beyond $\mathrm{BiVO}_{4}$, and is a potentially generalizable phenomenon.

Recently, Favaro et al. ${ }^{17}$ have reported the formation of a $\mathrm{Bi}_{x}\left(\mathrm{PO}_{4}\right)_{y}$ layer at the surface of a $\mathrm{BiVO}_{4}$ photoanode when illuminated in a phosphate buffer under open-circuit conditions. A similar copper borate layer was formed during the examination of $\mathrm{CuWO}_{4}$ when it was photocharged in a borate buffer. ${ }^{14}$ These results suggest that an overlayer is formed at these metal oxide photoanode surfaces upon illumination by the chemisorption of the electrolyte anionic species. It was proposed that this semiconducting overlayer then creates a heterojunction with the underlying photoanode, improving the charge separation close to the surface and thus suppressing the surface recombination of the photoexcited charge carriers. To better explain the enhancements in charge separation and suppression of surface recombination of the photocharged electrodes, it is necessary to gain further insights into the modification of the electronic structure of the metal oxide photoanodes by this light-induced overlayer. This knowledge can help to improve the general understanding of an illuminated metal oxide/electrolyte interface, which is crucial for the development of PEC water splitting devices. In this work, $\mathrm{BiVO}_{4}$ is used as a model photoanode to study the changes in its electronic structure during photocharging in a borate electrolyte using a combination of X-ray photoelectron spectroscopy (XPS), ultraviolet-visible (UV-vis) spectroscopy, and ambient-pressure (in air) X-ray Raman scattering (XRS) spectroscopy. XPS only penetrates the first $\sim 5 \mathrm{~nm}$ of the $\mathrm{BiVO}_{4}$ film and therefore provides surface-sensitive information, while both UV-vis and XRS techniques provide bulk-sensitive information. A surface hydroxide layer was found to form spontaneously even when the $\mathrm{BiVO}_{4}$ electrode was placed in the electrolyte in the dark. A bismuth borate layer was formed under the photocharging conditions, creating a heterojunction that resulted in an improved charge separation and decreased charge carrier recombination near the surface as discovered through $\mathrm{ab}$ initio simulations of the $\mathrm{O} \mathrm{K}$ edge spectra of $\mathrm{BiVO}_{4}$. These findings validate the previous hypotheses proposed in the literature, ${ }^{14,17}$ about improved band bending in the space charge region (SCR) as a result of the light-induced heterojunction formation. These results highlight the complex and dynamic nature of metal oxideelectrolyte interfaces and give new insights into the mechanisms for PEC performance enhancement of photocharged $\mathrm{BiVO}_{4}$ photoanodes. More importantly, this work highlights that the surface adsorption of anionic electrolyte species on metal oxide surfaces cannot be ignored, as it has serious implications on the fundamental understanding and performance analysis of metal oxide photoanodes.

\section{EXPERIMENTAL SECTION}

Fabrication of $\mathrm{BiVO}_{4}$ Thin-Film Photoanodes. Thin films of $\mathrm{BiVO}_{4}$ were deposited on fluorine-doped tin oxide (FTO) substrates via spray pyrolysis. The details of the experimental procedure can be found elsewhere. ${ }^{18}$ In short, FTO substrates were first coated with $\sim 80 \mathrm{~nm}$ of $\mathrm{SnO}_{2}$ while the substrate temperature was kept at $425^{\circ} \mathrm{C}$, and subsequently coated with $200 \mathrm{~nm}$ of $\mathrm{BiVO}_{4}$ while the substrate was kept at $450{ }^{\circ} \mathrm{C}$. The samples were then annealed in an air-flushed tube furnace for $2 \mathrm{~h}$ at $460{ }^{\circ} \mathrm{C}$.

X-ray Raman Scattering Spectroscopy. All X-ray Raman scattering spectroscopy data were gathered using the dedicated large solid angle spectrometer at the ID20 beamline of the European Synchrotron Radiation Facility. ${ }^{19}$ These experiments were performed in air under atmospheric conditions. A pink beam from four U26 undulators was monochromatized to a $9686 \mathrm{eV}$ elastic energy, first by using a cryogenically cooled $\mathrm{Si}(111)$ monochromator and then a $\mathrm{Si}(311)$ Channel Cut postmonochromator. The beam was focused using a mirror system in Kirkpatrick-Baez geometry yielding a spot size of approximately $50 \times 100 \mu \mathrm{m}^{2}(\mathrm{~V} \times \mathrm{H})$ at the sample position. Given the experimental setup, the signal coming from 36 spherically bent $\operatorname{Si}(660)$ analyzer crystals in the vertical scattering plane was exploited. The overall energy resolution was $0.7 \mathrm{eV}$, and the mean momentum transfer was $6.2 / \mathrm{pm} 0.4 / \AA^{-1}$.

The reference sample used for this study was a $\mathrm{BiVO}_{4}$ film prepared as described above and measured without any further treatment at a $0.5^{\circ}$ angle relative to the incidence beam (penetration depth, $60 \mathrm{~nm}$ ). Pellets of cellulose and vanadium oxide $\left(\mathrm{V}_{2} \mathrm{O}_{4}\right.$ and $\mathrm{V}_{2} \mathrm{O}_{5}$, SigmaAldrich) were also measured at a $0.5^{\circ}$ angle. A grazing incidence angle is required to ensure surface-sensitive measurements when a hard Xray beam is used. Before the XRS measurements, the dark and PC samples were kept in open-circuit conditions in a flow cell with a 0.1 $\mathrm{M}$ sodium borate buffer (sodium tetraborate decahydrate (SigmaAldrich, 99.5\%) in Milli-Q water, while $\mathrm{NaOH}$ was added until $\mathrm{pH} 10$ was reached) for $12 \mathrm{~h}$ in the dark and under illumination, respectively. The flow cell was further equipped with a glass $\mathrm{Ag} / \mathrm{AgCl}$ reference electrode (XR300, Radiometer Analytical) and a coiled Pt wire as counter electrode both in the dark (dark sample) and under illumination (photocharged sample, $100 \mathrm{~mW} / \mathrm{cm}^{2}$ Xe lamp with water filter). After $12 \mathrm{~h}$, the electrodes were removed from the electrochemical cell, quickly dried under a flow of nitrogen, and moved to the experimental hutch for the XRS measurements. The preparation procedure was performed very quickly to ensure the XRS measurements could start less than $15 \mathrm{~min}$ after the sample was removed from the electrochemical cell. During the XRS measurements, the incident energy was scanned at a fixed analyzer energy of $9.7 \mathrm{keV}$ to create energy losses in the vicinity of the core-electron excitations of interest $\left(\mathrm{L}_{2,3}\right.$ of $\mathrm{V}$ and $\mathrm{K}$ edge of $\left.\mathrm{O}\right)$. The data collected by three pixelated Maxipix detectors ${ }^{20}$ were integrated over appropriated regions of interest, then averaged over the $36 \mathrm{Si}(660)$ analyzer crystals, and treated with the XRS-tools program package. A background accounting for the valence Compton profile was subtracted as described elsewhere. ${ }^{21}$ Finally, a normalization over an area of $40 \mathrm{eV}$ across the edge was applied.

Eight energy loss scans per sample were measured at room temperature, each one at a new sample position to avoid beam radiation damage. All XRS measurements were checked for consistency prior to summation to rule out radiation damage of the sample induced by the X-ray beam.

$\mathrm{Ab}$ initio XRS simulations at the $\mathrm{O} \mathrm{K}$ edge were performed with the finite difference method near-edge structure (FDMNES) software package. $^{22,23}$ The calculated spectra were compared to both photocharged and dark samples. The input parameter files of the calculations are reported in the Supporting Information (SI). The 
vanadium $\mathrm{L}$ edges were simulated with $\mathrm{Crispy}^{24}$ using a multiplet analysis approach.

X-ray Photoelectron Spectroscopy. XPS spectra were obtained using a Thermo Scientific K-Alpha apparatus equipped with an $\mathrm{Al} \mathrm{K}$ Alpha X-ray source and a Flood Gun for charge compensation of the sample. Parameters used for the measurements were: spot size, 400 $\mu \mathrm{m}$; pass energy, $50 \mathrm{eV}$; energy step size, $0.1 \mathrm{eV}$; dwell time, $50 \mathrm{~ms}$; 10 scans in the vicinity of the Bi $4 \mathrm{f}, \mathrm{C} 1 \mathrm{~s}, \mathrm{Na} 1 \mathrm{~s}, \mathrm{O} 1 \mathrm{~s}$, and V $2 \mathrm{p}$ orbital binding energy with alternative scan numbers for B 1 s (200 scans) and the valence band (50 scans). Samples were rinsed and dried before measuring.

UV-Vis Spectroscopy. UV-vis transmission measurements were performed on the reference, dark, and the photocharged $\mathrm{BiVO}_{4}$ samples using a PerkinElmer Lambda 900 UV/Vis/NIR spectrometer. The transmission measurements were performed inside an integration sphere in the transmittance mode $(\% T)$. Data were recorded with a $15^{\circ}$ tilt in the sample, with respect to the incident light beam to minimize reflection. A scan rate of $250 \mathrm{~nm} / \mathrm{min}$ and an integration time of $0.2 \mathrm{~s}$ were used for the data capture. The absorption coefficient and the Tauc plot were calculated from the transmittance data using the formulas in the SI.

High-Resolution Scanning Electron Microscopy (HR-SEM). HR-SEM images were taken with a Nova NanoSEM at an accelerating voltage of $10 \mathrm{kV}$.

\section{RESULTS}

To gain further insights into the mechanisms of the photoelectrochemical enhancement that arise from the photocharging procedure in $\mathrm{BiVO}_{4}$, several material properties of $\mathrm{BiVO}_{4}$ were investigated before and after photocharging. In particular, the influence of placing the photoanodes in solution under open-circuit conditions with and without illumination was examined. A reference $\mathrm{BiVO}_{4}$ sample (as-deposited) was compared to both a $\mathrm{BiVO}_{4}$ film that was kept under opencircuit conditions and AM1.5 light illumination (i.e., photocharged) for $12 \mathrm{~h}$ and $\mathrm{BiVO}_{4}$ film that was kept in open circuit for $12 \mathrm{~h}$ in the dark. The samples are referred to as untreated $\mathrm{BiVO}_{4}$, photocharged $\mathrm{BiVO}_{4}\left(\mathrm{PC}-\mathrm{BiVO}_{4}\right)$, and dark $\mathrm{BiVO}_{4}$, respectively. The samples were studied through several spectroscopic techniques, including UV-vis spectroscopy, XPS, and XRS. Both XPS and XRS give electronic and structural information on the film; however, due to the difference in penetrating depth of the X-rays in the different experiments, XPS gives information about the surface of the films while XRS provides information on the structure up to 60 $\mathrm{nm}$ into the bulk of $\mathrm{BiVO}_{4}$.

X-ray Photoelectron Spectroscopy. XPS measurements were performed on reference, dark, and $\mathrm{PC}-\mathrm{BiVO}_{4}$ films, and the results are shown in Figure 1. The presented spectra are background-subtracted and energy-corrected with the $\mathrm{C} 1 \mathrm{~s}$ signal (Figure S1d). Several trends can be observed between the different samples. The Bi $4 \mathrm{f}$ peaks are shown in Figure 1a, and a shift was observed from $159.5 \mathrm{eV}$ for the reference sample to 159.0 and $158.7 \mathrm{eV}$ for the dark and photocharged $\mathrm{BiVO}_{4}$, respectively. This shift is accompanied by slight broadening of the $\mathrm{Bi} 4 \mathrm{f}$ peaks for the dark and the photocharged samples, as shown with the increase in full width at half-maximum in Table S1. A signal from the B 1s spectrum is not present in the reference sample, while it appears very small in the dark and is much more pronounced in the $\mathrm{PC}_{-} \mathrm{BiVO}_{4}$, as seen in Figure $1 \mathrm{~b}$. In Figure 1c, the main signal from the oxygen ( $\mathrm{O} 1 \mathrm{~s}$ ) spectra was observed to decrease slightly in binding energy from $530.3 \mathrm{eV}$ for the reference sample to 529.9 and $529.6 \mathrm{eV}$ for the dark and PC$\mathrm{BiVO}_{4}$ samples, respectively, while the shoulder peak at $531 \mathrm{eV}$
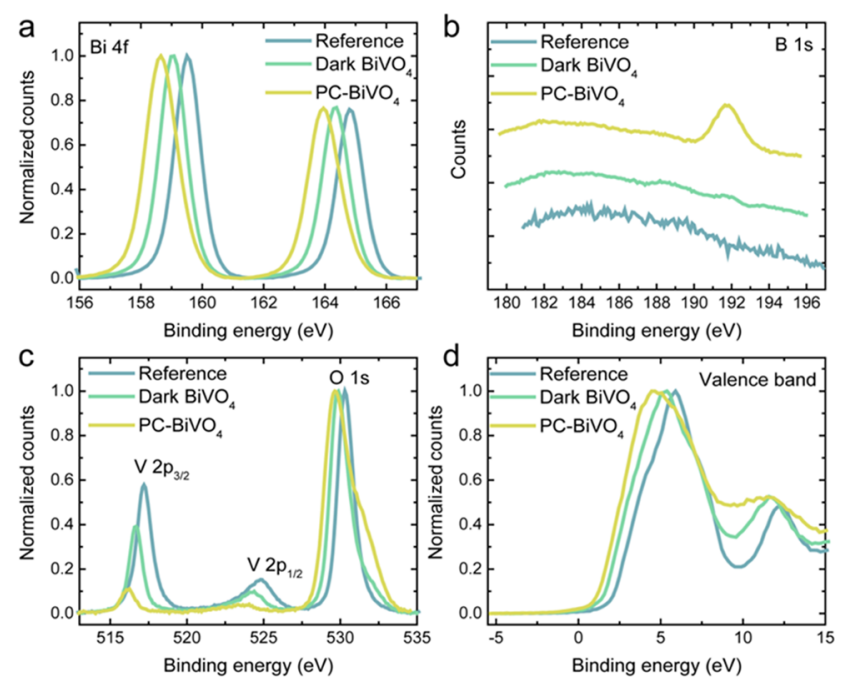

Figure 1. XPS spectra of the reference $\mathrm{BiVO}_{4}$ and the dark and PC$\mathrm{BiVO}_{4}$ samples after $22 \mathrm{~h}$ under open-circuit conditions in a borate buffer electrolyte. Spectra of (a) Bi 4f, (b) B 1s, (c) V 2p and O 1s, and (d) the valence band are shown.

grows significantly (deconvolution of the $\mathrm{O} 1 \mathrm{~s}$ peaks is given in Figure S1). The peak signal from the V 2 p spectra is shown in Figure $1 c$, where a shift to lower binding energies is observed along with a strong signal decrease when looking at the reference, dark, and $\mathrm{PC}-\mathrm{BiVO}_{4}$ films, respectively.

When these results are compared to prior literature, several important similarities can be seen. Favaro et al. found a bismuth phosphate layer forming on the surface of the $\mathrm{BiVO}_{4}$ films under illumination that slowly disappeared again after the light was turned off. ${ }^{17}$ In our current work, the buffer used is not a phosphate but rather a borate electrolyte. The binding energies of $\mathrm{Bi}$ to borate and phosphate are not the same, which implies that the peak shift found in our $\mathrm{Bi} 4 \mathrm{f}$ spectrum is not the same as in the work by Favaro. A previous report on $\mathrm{B}_{2} \mathrm{O}_{3}-\mathrm{Bi}_{2} \mathrm{O}_{3}$ glasses reported the $\mathrm{Bi} 4 \mathrm{f}$ binding energies of $\mathrm{Bi}-$ $\mathrm{O}-\mathrm{Bi}$ at $159.85 \mathrm{eV}$ and $\mathrm{B}-\mathrm{O}-\mathrm{Bi}$ at $158.7 \mathrm{eV}$, which was explained by the presence of boron shifting the $\mathrm{Bi}$ signal to lower binding energies. ${ }^{25}$ In addition, the $\mathrm{O} 1 \mathrm{~s}$ binding energies were reported to be $530.35 \mathrm{eV}$ for $\mathrm{Bi}_{2} \mathrm{O}_{3}$ and 530.95 $\mathrm{eV}$ for $\mathrm{B}_{2} \mathrm{O}_{3}$. In our work, we find a shoulder in the $\mathrm{O} 1 \mathrm{~s}$ spectra at $531.0 \mathrm{eV}$ to increase during photocharging and coincide with the emergence of the $B 1 \mathrm{~s}$ peak at $192.0 \mathrm{eV}$ (Figures 1b,c, and S1). We therefore ascribe the shift and broadening of the $\mathrm{Bi} 4 \mathrm{f}$ peak to the emergence of the $\mathrm{O} 1 \mathrm{~s}$ shoulder together with the B 1 s peak to reveal the formation of a surface bismuth borate layer during photocharging. This surface borate layer is very similar to the phosphate layer found by Favaro et al. In addition to the XPS data given for Bi, B, and $\mathrm{O}$, we also observed a shift toward lower binding energies for the $\mathrm{V} 2 \mathrm{p}$ signal, which indicates a reduction in the oxidation state of the vanadium. ${ }^{11}$

When considering the difference between the dark and photocharged samples, it is interesting to note that the electronic features of the dark sample show an intermediate phase between the photocharged and the reference sample. Although the dark sample has no clear B 1s signal, it still has a partial shoulder in the $\mathrm{O} 1 \mathrm{~s}$ spectrum, and it is likely that this sample instead has a surface coverage of bismuth hydroxide as previously reported. ${ }^{11}$ Since the binding energies for $\mathrm{Bi}-\mathrm{O}-\mathrm{B}$ and $\mathrm{Bi}-\mathrm{O}-\mathrm{H}$ are almost identical (the electronegativity values 

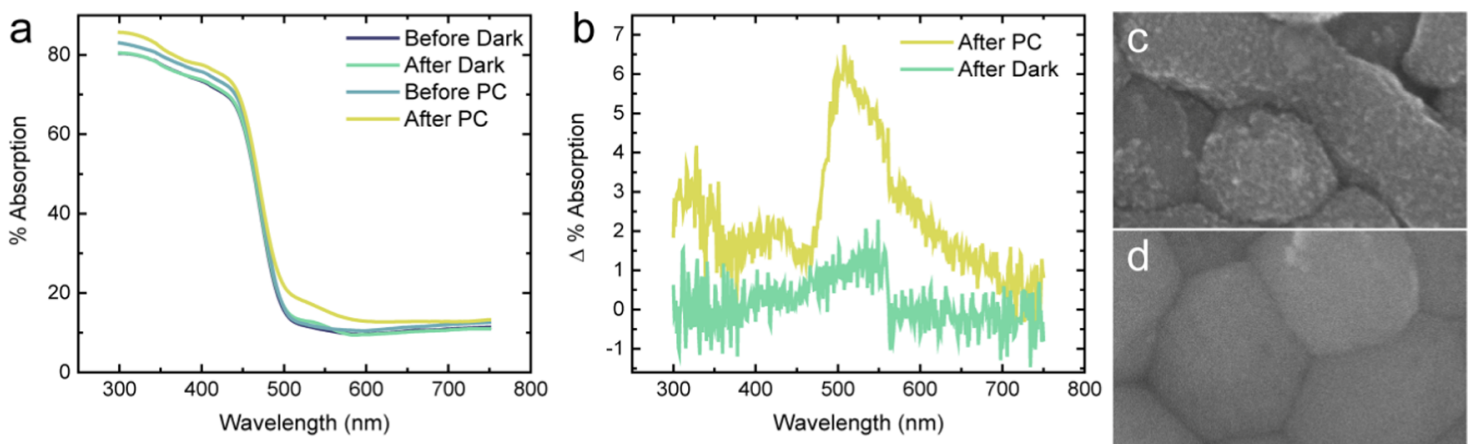

Figure 2. (a) UV-vis absorption spectra of a dark, before (purple) and after (green) the treatment and photocharged before (blue) and after (yellow) the treatment. (b) Difference spectra between before and after showing a defect-related feature in the PC sample. (c) HR-SEM image of PC-BiVO 4 showing small particles (white spots), finely dispersed over the $\mathrm{BiVO}_{4}$ grains. (d) HR-SEM image of dark BiVO . Width of HR-SEM images is $520 \mathrm{~nm}$.

of $\mathrm{H}$ and $\mathrm{B}$ are 2.2 and 2.0 , respectively), the distinction is difficult to make without analyzing the $\mathrm{B}$ 1s signal as well.

The valence band (VB) of the reference, dark, and PC$\mathrm{BiVO}_{4}$ electrodes was also examined, and is shown in Figure 1d. It can be seen from this figure that similar to the previously observed shifts in the binding energy of $\mathrm{Bi}, \mathrm{O}$ and $\mathrm{V}$, there is also a shift in the valence band energy toward lower binding energies when looking at the reference, dark, and $\mathrm{PC}-\mathrm{BiVO}_{4}$ samples, respectively.

A broadening to lower binding energy of the valence band spectra of the dark and $\mathrm{PC}-\mathrm{BiVO}_{4}$ is also visible, due to the convolution of the VB spectra of bismuth hydroxide/bismuth borate and bismuth vanadate, respectively. These results indicate that the bismuth borate layer formed on the surface has a valence band closer to the Fermi level of the composite system. The exact composition of the bismuth borate surface layer is unknown, but from prior work on bismuth borate glasses, we can estimate the band gap of the bismuth borate layer to be equal to or larger than that of $\mathrm{BiVO}_{4}{ }^{20,26}$

The unidirectional peak shifts in the $\mathrm{Bi} 4 \mathrm{f}, \mathrm{V} 2 \mathrm{p}, \mathrm{O} 1 \mathrm{~s}$, and VB XPS spectra to lower binding energies are in part an electronic effect. Band bending caused by the equilibration of the Fermi level of $\mathrm{BiVO}_{4}$ and the chemisorbed anionic surface layer shifts the XPS spectra. Similar XPS peak shifts were observed by Hermans et al. ${ }^{27}$ where different metal oxide surface layers were deposited on $\mathrm{BiVO}_{4}$, and the peak shifts were attributed to a change in Fermi level position because of the surface layer-induced band bending. $\mathrm{NiO}$ and $\mathrm{CoO}_{x}$ surface layers lead to an upward band bending, whereas indium-doped tin oxide induced a downward band bending in $\mathrm{BiVO}_{4}$, due to the differences in their respective work functions compared to that of $\mathrm{BiVO}_{4}$. The core-level XPS spectra shifted to lower binding energies with the deposition of $\mathrm{NiO}$ and $\mathrm{CoO}_{x}$ overlayers, similar to the shifts seen with dark and PC$\mathrm{BiVO}_{4}$ in this study. Note that band bending-induced shifts should be equal in magnitude for different core levels. Table S2 shows that the shifts for $\mathrm{V}$ and $\mathrm{Bi}$ are larger than those for $\mathrm{O}$, meaning that the $\mathrm{V}$ and $\mathrm{Bi}$ shifts also have a chemical component to them, assigned above as a reduction in $\mathrm{V}$ oxidation state and the appearance of the $\mathrm{Bi}$ borate surface layer.

The formation of the bismuth borate layer at the surface forms a heterojunction with the $\mathrm{BiVO}_{4}$ which induces an upward band bending and reduces photogenerated charge recombination at the semiconductor-liquid junction. The cathodic Fermi level shift that was observed with the open- circuit potential measurements for the dark and photocharged samples, as shown in Figure S2, is another evidence of the band bending observed through the XPS shifts.

Ultraviolet-Visible Spectroscopy. UV-vis spectroscopy was performed before and after the photocharging process to determine if there were any optical changes in the $\mathrm{BiVO}_{4}$ films during the treatment that may arise from the formation of the bismuth borate surface layer. In Figure $2 a$, the spectra of a dark (green) and photocharged (yellow) sample are shown together with their corresponding spectra before treatment. A small increase in absorption is found within the band gap of $\mathrm{BiVO}_{4}$ for the photocharged sample, while the dark sample does not show this increase in absorption. Just outside the band gap energy, between 475 and $700 \mathrm{~nm}$, the photocharged sample shows a very distinct absorption feature.

The absorption increase inside the band gap could explain

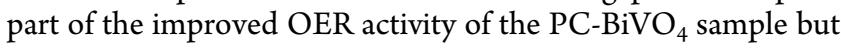
cannot account for the observed 3-fold increase in the incident photon-to-current conversion efficiency. ${ }^{11}$ To determine whether the choice of buffer anion has any effect on this absorption feature, photocharging was performed in a phosphate buffer at $\mathrm{pH} 10$. This treatment revealed a very similar improvement within the absorption spectra after photocharging (Figure S3a). When subtracting the spectrum of the before-PC from the after-PC spectrum (Figure 2b), a feature emerges that resembles either a surface plasmon resonance mode with a peak in absorption around $520 \mathrm{~nm}$ or a

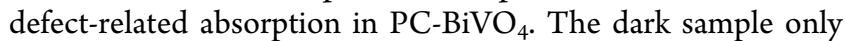
has a minor increase in this region, but still shows a feature with a peak centered around $520 \mathrm{~nm}$. Plasmonic features of vanadium oxide are only found in the infrared region, ${ }^{28}$ and those for metallic vanadium are found in the UV region, ${ }^{29}$ whereas metallic bismuth nanoparticles in an oxide medium can give rise to plasmonic features around $500 \mathrm{~nm} .^{20,30}$ However, the $\mathrm{Bi} 4 \mathrm{f}$ XPS spectra do not show any indication of the presence of metallic bismuth in the $\mathrm{PC}-\mathrm{BiVO}_{4}$. Therefore, we tentatively assign this feature to defect-related light absorption in $\mathrm{PC}^{-\mathrm{BiVO}_{4}}$. In fact, such above band gap optical features have been observed with some metal oxides ${ }^{31,32}$ and have often been attributed to defect-related light absorption, induced by surface oxygen vacancies. Therefore, the anion adsorption on the $\mathrm{BiVO}_{4}$ surface upon photocharging could have introduced surface oxygen vacancies through surface restructuring. A high-resolution scanning electron microscopy

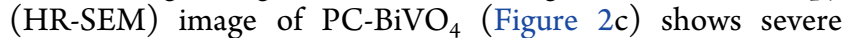
roughening of the surface on the nanoscale, compared to the 
dark and reference samples (Figures $2 \mathrm{~d}$ and S3c, respectively). This surface roughening could be a result of the surface restructuring to accommodate the buffer anion adsorption.

X-ray Raman Scattering. To gain further insight into the structural and electronic changes induced by the photocharging treatment, the vanadium $\mathrm{L}_{2}$ and $\mathrm{L}_{3}$ edge and the oxygen $\mathrm{K}$ edge were probed using $\mathrm{X}$-ray Raman scattering spectroscopy in near in situ conditions. X-ray absorption spectroscopy uses soft X-rays to probe light elements $(<1$ $\mathrm{keV})$; however, these $\mathrm{X}$-rays require ultra-high-vacuum conditions and are therefore less suitable for in situ studies. Instead, XRS allows the use of hard X-rays to probe elements with core-electron excitation energies in the soft X-ray regime, i.e., lower than $1 \mathrm{keV}$. The use of hard X-rays leads to a bulksensitive approach and can offer the possibility of working under operando conditions. In this work, we were able to study the $\mathrm{BiVO}_{4}$ structure at room temperature and pressure and measure spectra immediately after the photocharging treatment was performed. Real operando conditions would greatly complicate the analysis of the $\mathrm{O} \mathrm{K}$ edge spectrum since the electrolyte also contains oxygen, instead this near in situ approach was adopted. A grazing incidence angle was maintained to avoid contributions from the oxygen-containing FTO substrate and to increase the footprint of the X-ray beam on the sample. The vanadium $\mathrm{L}_{3}$ and $\mathrm{L}_{2}$ edges and oxygen $\mathrm{K}$ edge can be probed within the same measurement since their absorption energies are very close. The electronic structure of the various $\mathrm{BiVO}_{4}$ samples is obtained from XRS data by simulating the data with ab initio-based calculations.

The effect of photocharging on the electronic states in the conduction band of $\mathrm{BiVO}_{4}$ was studied by comparing a photocharged (PC) sample and a "dark" sample to a reference sample. To try and preserve the operando state, the samples were not rinsed but only dried before being transferred to the XRS measurement chamber. All samples were fabricated according to the same procedure, and XRS measurements were taken under identical settings. The XRS spectra of the three samples are shown together in Figure 3.

The region from 515 to $545 \mathrm{eV}$ covers the core-electron excitations of interest, namely, the vanadium $\mathrm{L}_{2}$ and $\mathrm{L}_{3}$ and oxygen $\mathrm{K}$ edges. For low transferred momenta, the orbital selection rule dictates $\Delta l= \pm 1$, where $l$ is the azimuthal quantum number. Therefore, the vanadium $L$ edge excitation

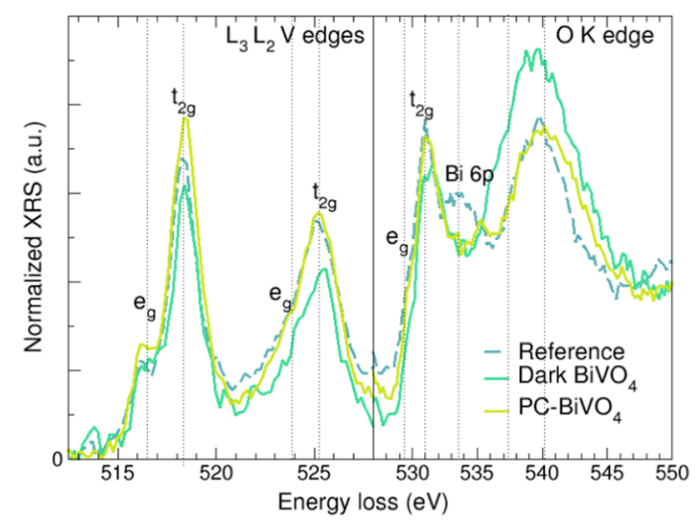

Figure 3. Normalized XRS spectra of the vanadium $\mathrm{L}_{2}, \mathrm{~L}_{3}$, and the oxygen $\mathrm{K}$ edge region of a reference, dark, and $\mathrm{PC}-\mathrm{BiVO}_{4}$ sample. The changing ratio of the $\mathrm{O} \mathrm{K}$ edge peaks reveals a filling with electrons of the pre-edge peak $\left(\mathrm{O} 2 \mathrm{p}-\mathrm{V} 3 \mathrm{~d} \mathrm{e}_{\mathrm{g}} / \mathrm{t}_{2 \mathrm{~g}}\right)$ for the dark sample. results in a transition to the first available vanadium $d$ orbital and the oxygen $\mathrm{K}$ edge excitation transitions to the first unoccupied oxygen $\mathrm{p}$ orbital.

The XRS spectrum of the reference thin-film $\mathrm{BiVO}_{4}$ sample (dashed blue line in Figure 3) is discussed first. Four main peaks with shoulders to the left of the first three peaks are easily discernible. The first main peak between 515 and $520 \mathrm{eV}$ corresponds to the $\mathrm{V} \mathrm{L}_{3}$ edge: the excitation from $\mathrm{V} \mathrm{2} \mathrm{p}_{3 / 2}$ to conduction band V 3d states; the second main peak from 521 to $528 \mathrm{eV}$ is the $\mathrm{V} \mathrm{L}_{2}$ edge: the excitation from $2 \mathrm{p}_{1 / 2}$ to the conduction band $\mathrm{V} 3 \mathrm{~d}$ states. ${ }^{33}$ The $\mathrm{L}_{3}$ region consists of a main peak at $518 \mathrm{eV}$ and a shoulder at $516 \mathrm{eV}$, which are the $\mathrm{t}_{2 \mathrm{~g}}$ (main peak) and $\mathrm{e}_{\mathrm{g}}$ (left shoulder) states of the $\mathrm{VO}_{4}$ structure within the $\mathrm{BiVO}_{4}$ lattice. ${ }^{33,34}$ The $\mathrm{V} 3 \mathrm{~d}$ orbitals are hybridized with the $\mathrm{O} 2 \mathrm{p}$ orbital. The $\mathrm{L}_{2}$ edge is excited to the same (hybridized) V $3 \mathrm{~d} \mathrm{t}_{2 \mathrm{~g}}$ and $\mathrm{e}_{\mathrm{g}}$ states; however, this part of the spectrum is noisier than the $\mathrm{V} \mathrm{L}_{3}$ edge because the core hole lifetime at the $\mathrm{L}_{2}$ edge is shorter resulting in a broader spectrum. All excited-state energies that are visible in Figure 3 are given in Table 1 with their corresponding orbital

Table 1. All Assigned Peaks of the Reference $\mathrm{BiVO}_{4}$ Sample as Depicted in Figure 3

\begin{tabular}{|c|c|c|}
\hline probed edge & energy $(\mathrm{eV})$ & electronic transition \\
\hline \multirow[t]{3}{*}{$\mathrm{V} \mathrm{L}_{3}$} & & from $2 \mathrm{p}_{3 / 2}$ to unoccupied $V 3 \mathrm{~d}$ states \\
\hline & 516 & to $\mathrm{e}_{\mathrm{g}} \mathrm{V} 3 \mathrm{~d}-\mathrm{O} 2 \mathrm{p}\left(\mathrm{d}_{x^{2}-y^{2}}, \mathrm{~d}_{z^{2}}\right)$ \\
\hline & 518 & 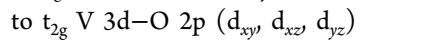 \\
\hline \multirow[t]{3}{*}{$\mathrm{V} \mathrm{L}_{2}$} & & from $2 \mathrm{p}_{1 / 2}$ to unoccupied $V 3 \mathrm{~d}$ states \\
\hline & 523 & 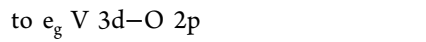 \\
\hline & 525 & to $t_{2 g} V 3 d-O 2 p$ \\
\hline \multirow[t]{5}{*}{$\mathrm{O} \mathrm{K}$} & & from $\mathrm{O} 1 \mathrm{~s}$ to unoccupied $\mathrm{O} 2 \mathrm{p}$ states \\
\hline & 530 & to $e_{g} V 3 d-O 2 p$ \\
\hline & 532 & to $t_{2 g} V 3 d-O 2 p$ \\
\hline & 534 & to $\pi^{*} \mathrm{Bi} 6 \mathrm{p}-\mathrm{O} 2 \mathrm{p}$ \\
\hline & 540 & to $\sigma^{*}$ from $\mathrm{V} 4 \mathrm{~s}$ and $\mathrm{Bi} 6 \mathrm{~s}$ with $\mathrm{O} 2 \mathrm{p}$ \\
\hline
\end{tabular}

transitions. Overall, the XRS spectra shows good agreement with soft X-ray absorption near-edge structure (XANES) spectra of $\mathrm{BiVO}_{4}$ in the literature. ${ }^{33,34}$

The oxygen $\mathrm{K}$ excitations extend from $528 \mathrm{eV}$ upward and consist of transitions from the occupied $\mathrm{O} 1 \mathrm{~s}$ orbital close to the core of the oxygen atom to the unoccupied $\mathrm{O} 2 \mathrm{p}$ states. A peak at $531 \mathrm{eV}$ and its shoulder at $530 \mathrm{eV}$ show the $\mathrm{O} 2 \mathrm{p}-\mathrm{V}$ $3 \mathrm{~d}$ hybridization and thus represent the same orbitals as the $\mathrm{V}$ $\mathrm{L}_{2}$ and $\mathrm{L}_{3}$ edge spectrum. Therefore, again these peaks are split into the $e_{g}$ and $t_{2 g}$ states according to the tetrahedral crystal field splitting. ${ }^{33}$ A second strong peak in the spectrum at 534 $\mathrm{eV}$ corresponds to antibonding $\pi \mathrm{O} 2 \mathrm{p}-\mathrm{Bi} 6 \mathrm{p}$ states. $^{34,35}$ The final peak at $540 \mathrm{eV}$ is the excitation to antibonding states of $\sigma$ character originating from the hybridization of both $\mathrm{V} 4 \mathrm{~s}$ and $\mathrm{Bi} 6 \mathrm{~s}$ with $\mathrm{O} 2 \mathrm{p} .{ }^{33,36}$ Since the hybridization between $\mathrm{V}$ and $\mathrm{O}$ is stronger than that between $\mathrm{Bi}$ and $\mathrm{O}$, the $\mathrm{O} \mathrm{K}$ edge spectra mainly consist of $\mathrm{V}-\mathrm{O}$ states. $^{36}$

According to Cooper et al., the hybridized V 3d-O 2p orbitals are not simply split into the $e_{g}$ and $t_{2 g}$ tetrahedral molecular orbitals; instead, three different V $3 \mathrm{~d}$ contributions should be present in the spectrum. ${ }^{34}$ This is due to the fact that the monoclinic scheelite form of $\mathrm{BiVO}_{4}$ has lattice distortions causing the tetrahedral and dodecahedral symmetries to be broken. In fact, two separate $\mathrm{V}-\mathrm{O}$ bond lengths and four separate $\mathrm{Bi}-\mathrm{O}$ bond lengths are reported. ${ }^{36}$ Therefore, the $\mathrm{VO}_{4}$ has a $\mathrm{C}_{2}$ symmetry, leading to triplet splitting of the $\mathrm{V} 3 \mathrm{~d}$ 

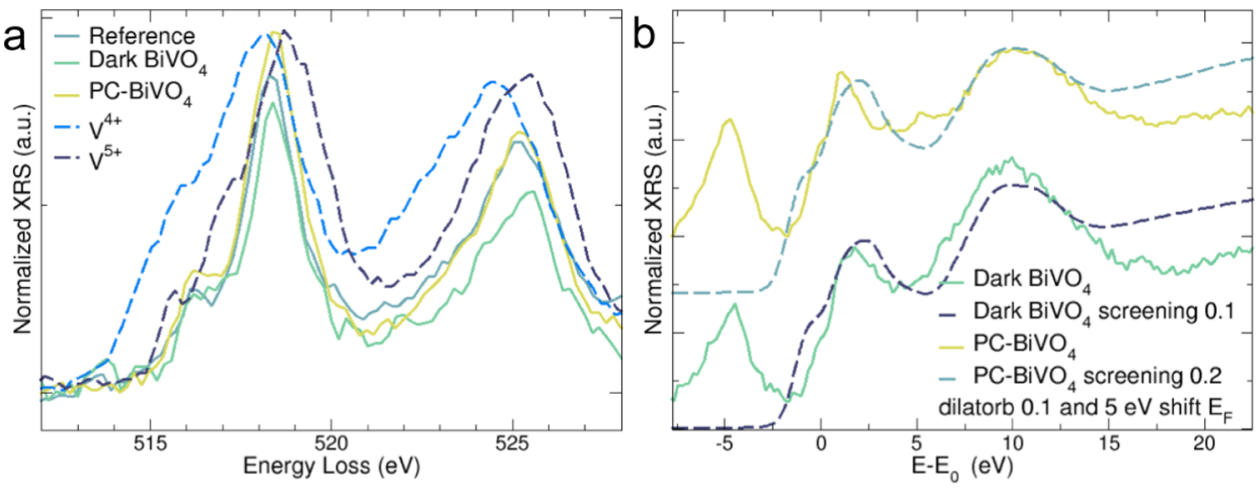

Figure 4. (a) XRS spectra of the vanadium oxides $\left(\mathrm{V}_{2} \mathrm{O}_{4}\right.$ and $\left.\mathrm{V}_{2} \mathrm{O}_{5}\right)$ are presented together with the reference, dark and $\mathrm{PC}$ - $\mathrm{BiVO}$ to show the effect of changing $\mathrm{V}$ oxidation state on the peak position in the $\mathrm{V} \mathrm{L}_{2}$ and $\mathrm{L}_{3}$ edge spectra. (b) The $\mathrm{O} \mathrm{K}$ edge spectra of the dark and PC sample are overlaid with $\mathrm{ab}$ initio simulations including the semiempirical dilatorb and screening parameters.

orbitals. This is not easily discernible from the measured spectrum because of the lower resolution of XRS compared to standard soft X-ray XANES. However, it is important to realize that the broad peaks observed are composed of overlapping contributions of several orbitals.

$\mathrm{XRS}$ spectra of $\mathrm{V}^{4+}$ and $\mathrm{V}^{5+}$ reference samples were collected to help interpret the oxidation state of $\mathrm{V}$ in $\mathrm{BiVO}_{4}$. These reference spectra were plotted together with those of reference, dark, and photocharged $\mathrm{BiVO}_{4}$ and are presented in Figure 4a. As is evident from the figure, the peaks of the $\mathrm{V}^{4+}$ reference sample (dark blue) are shifted to lower binding energies compared to the peaks of the $\mathrm{V}^{5+}$ sample (purple). This is in very good agreement with the other studies in the literature. ${ }^{37,38}$ Another interesting aspect is that the peaks in the $\mathrm{V}$ L edge spectra for the reference, dark, and $\mathrm{PC}-\mathrm{BiVO}_{4}$ are in between the $\mathrm{V}^{4+}$ and $\mathrm{V}^{5+} \mathrm{L}$ edge reference spectra. From this, it can be concluded that the spray-deposited $\mathrm{BiVO}_{4}$ has a mix of $\mathrm{V}^{4+}$ and $\mathrm{V}^{5+}$ oxidation states. In an ideal $\mathrm{BiVO}_{4}$ lattice, the vanadium species should have a $5+$ oxidation state. However, it is very common to have intrinsic defects in the structure, in the form of oxygen vacancies. These oxygen vacancies are responsible for the n-type character of monoclinic $\mathrm{BiVO}_{4}$. Due to these oxygen vacancies, to maintain charge neutrality, some vanadium species in the $\mathrm{BiVO}_{4}$ lattice could exist in the 4+ oxidation state. Rossell et al. ${ }^{39}$ quantified the vanadium oxidation state in monoclinic $\mathrm{BiVO}_{4}$ using energy loss spectroscopy and suggested that the surface vanadium ions predominantly exist in a $\sim 4+$ oxidation state, due to the presence of a significant amount of oxygen vacancies.

To better understand these results, it is important to consider the penetration depth of the performed XRS measurements. Due to the grazing incidence angle of $0.5^{\circ}$, only the first $60 \mathrm{~nm}$ are probed. Additionally, the spraydeposited $\mathrm{BiVO}_{4}$ is highly nanostructured and hence has a high surface-to-volume ratio. Therefore, the obtained vanadium $\mathrm{L}$ edge spectra for the reference, dark, and photocharged samples could be an average of the surface and bulk oxidation state of vanadium that fall between the $4+$ and $5+$ oxidation states. These results are in alignment with the XRD patterns that show a strong resemblance between the reference and photocharged samples, both having monoclinic scheelite $\mathrm{BiVO}_{4}$ features (Figure S4). ${ }^{40}$ The reference, dark, and photocharged $\mathrm{BiVO}_{4}$ have similar vanadium $\mathrm{L}$ edge peak positions. However, the dark and $\mathrm{PC}-\mathrm{BiVO}_{4}$ show a slightly different ratio in intensity between the $\mathrm{V} \mathrm{L}_{3}$ and $\mathrm{L}_{2}$ edges compared to the reference spectra. A multiplet analysis was performed to predict the shape of the $\mathrm{V} \mathrm{L}$ edge spectra for a vanadium species in its $\mathrm{V}^{4+}$ and $\mathrm{V}^{5+}$ oxidation state, in a hypothetical bismuth vanadium oxide compound. These results are shown in Figure S5 and suggest that there could be a decrease in the oxidation state of vanadium with an increase in the $\mathrm{L}_{3} / \mathrm{L}_{2}$ peak ratio. The $\mathrm{V} \mathrm{L}_{3} / \mathrm{L}_{2}$ peak ratio is indeed higher for the dark and $\mathrm{PC}-\mathrm{BiVO}_{4}$ compared to the reference (Table S3) and would suggest a decrease in the oxidation state of vanadium, possibly from the creation of additional oxygen vacancies at the surface. This would be in agreement with the above band gap absorption feature seen in the absorption spectra of the $\mathrm{PC}-\mathrm{BiVO}_{4}$ and with the shift in the V 2p XPS spectrum. However, the low resolution of the XRS technique coupled with the surface and bulk averaging of the XRS technique makes this comparison difficult. Screening of V 2p XPS spectra by the surface-adsorbed layer also makes the comparison of the V 2p XPS spectra difficult. Additional experiments will be needed to study and quantify the vanadium oxidation state change in the dark and the photocharged sample, which is out of the scope of this work.

A disappearance of the $\mathrm{O} 2 \mathrm{p}-\mathrm{Bi} 6 \mathrm{p}$ peak at $534 \mathrm{eV}$ was observed for both the PC sample and the dark sample. As mentioned previously, the $\mathrm{Bi}-\mathrm{O}$ hybridization is not very strong; therefore, a small change in the electronic structure can make the Bi $6 p$ signal less visible, which appears to be the case for the dark sample. Bismuth borate withdraws bismuth from $\mathrm{BiVO}_{4}$, which leads to the reduction of the $\mathrm{O} 2 \mathrm{p}-\mathrm{Bi} 6 \mathrm{p}$ signal in the XRS spectrum of the photocharged $\mathrm{BiVO}_{4}$. The broadening of the mixed metal $\mathrm{sp}-\mathrm{O} 2 \mathrm{p}$ peak implies the existence of multiple orbitals that partly overlap in energy. This can be caused by the presence of multiple $\mathrm{V}$ oxidation states that all have molecular orbitals of slightly different energy.

The main difference between the dark and $\mathrm{PC}-\mathrm{BiVO}_{4}$ XRS spectra is the filling with electrons of the $\mathrm{V} 3 \mathrm{~d}$ states of the dark sample, as observed from a difference in peak height ratio between the pre-edge peak $(\mathrm{V} 3 \mathrm{~d}-\mathrm{O} 2 \mathrm{p})$ and the main peak (mixed metal sp-O 2p) in the $\mathrm{O}$ K edge spectrum. This lowering of the pre-edge peak implies a filling of the $\mathrm{d}$ states, resulting in a higher concentration of electrons for the dark sample compared to the reference and photocharged samples. The reason for this difference is not immediately obvious, so to further elucidate and quantify the influence of the photocharging treatment on the electronic structure of $\mathrm{BiVO}_{4}, \mathrm{ab}$ initio simulations at the $\mathrm{O} \mathrm{K}$ edge were performed with the FDMNES program package. The calculated spectra were 

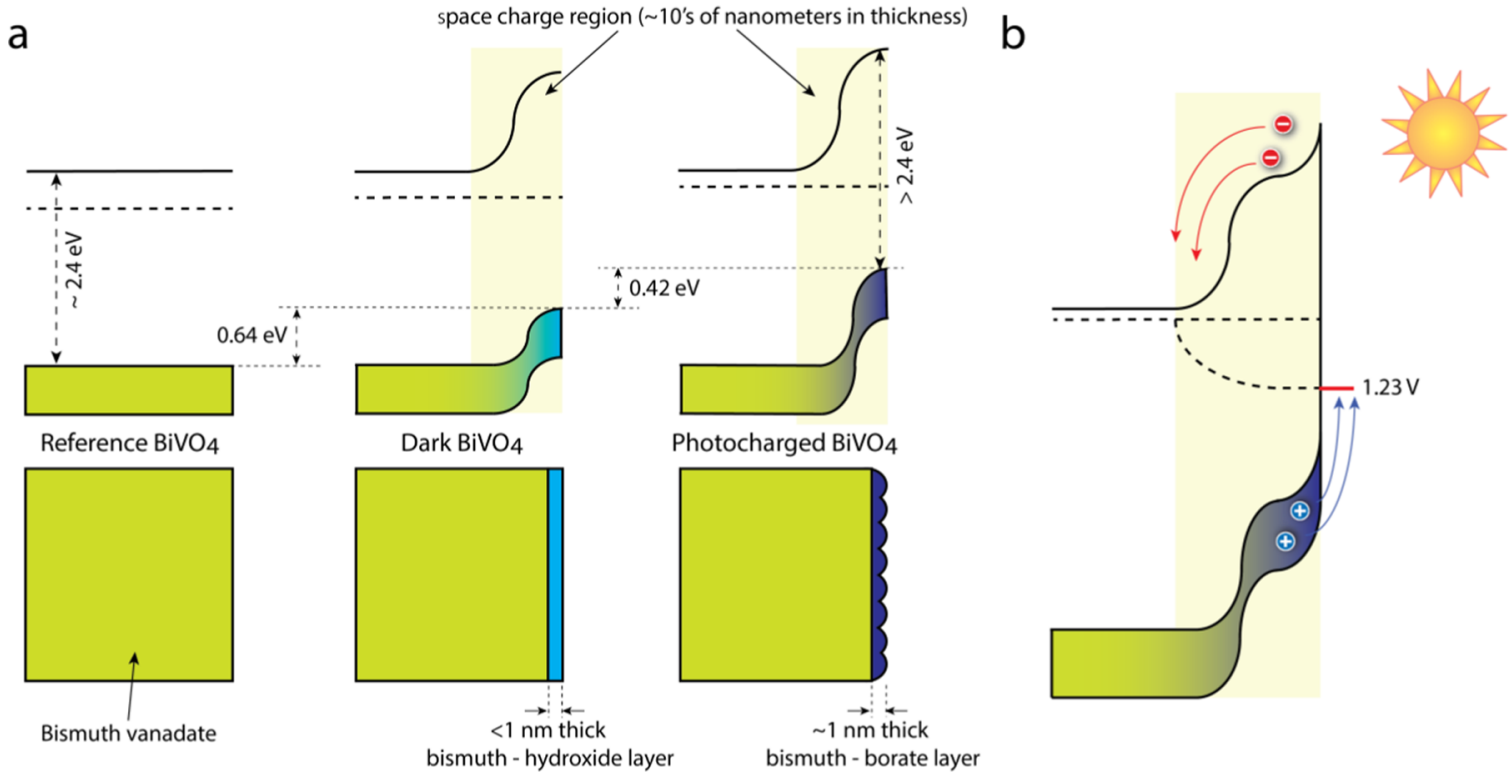

Figure 5. Effect of photocharging on the band gap region and the Fermi level of $\mathrm{BiVO}_{4}$. (a) Hydroxide (dark sample) and bismuth borate (PC sample) surface layers have a valence band closer to the Fermi level, introducing band bending at the surface. The band bending directs electrons

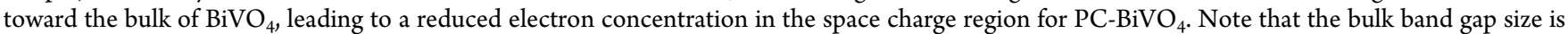
not altered by the PC treatment (Figure S10). (b) The effect of photocharging on $\mathrm{B} \mathrm{BiVO}_{4}$ photoanode in contact with an electrolyte under illumination. The additional band bending improves the charge separation, leading to the unrivaled $\mathrm{PEC}$ properties of $\mathrm{PC}^{-\mathrm{BiVO}_{4}}$.

compared to the experimentally obtained XRS spectra (the input parameter files of the calculations are shown in the SI). To simulate the peak height ratios observed in the experimental XRS spectra, two semi-empirical parameters, the dilatorb and screening parameters, were used in the calculations. The dilatorb and screening parameters provide the ability to tune the height of the pre-edge peaks and their position with respect to the main edge. Moreover, the dilatorb parameter provides the ability to consider the degree of ionicity of oxygen in the lattice structure by modifying the valence orbitals. Modifying this parameter means either dilating or contracting the valence orbitals, which is a necessary procedure when modeling anions with this package since the default is fully covalent, not considering the ionic character of oxygen. ${ }^{41}$ Anions have a more negative charge and are larger than their covalent radius because of the extra electrons that the anions take from the cations. The effect of changing the dilatorb parameter is shown in Figure S6.

The screening parameter is used to simulate a not fully screened core hole in the absorbing atom, meaning that part of the electron charge in the conduction band is missing. When the total excited electron is present in the conduction band, no charge is missing and the core hole is considered fully screened. The screening parameter generally points to a correlation effect caused by the presence of the excited $3 \mathrm{~d}$ electrons that hinder the screening process. In this respect, the screening value is an indication of the filling with electrons of the conduction band. However, oxygen electrons can behave unexpectedly to the removal of electrons, and it is therefore not always possible to predict the pattern in the $\mathrm{O} \mathrm{K}$ edge spectra as a function of electron depletion. Instead, the screening parameter can help making the trends of the spectra quantifiable. The effect of changing the screening parameter is shown in Figure S7.

If the effects of the dilatorb and the screening value are now combined, it is possible to effectively simulate the XRS spectra.
The best fitting simulations are shown in Figures 4 and S8. The reference spectrum (Figure S8) was fitted with a dilatorb value of 0.1 and no screening potential. The dark sample is fitted with a screening value of 0.1 and the dilatorb set to 0.1 . The photocharged sample, on the other hand, is fitted with a screening parameter of 0.2 and a dilatorb value of 0.1 . The default value for the screening parameter is 0 in the FDMNES software, so the lack of a defined screening value in the reference sample should be understood as full screening. The trend in the screening between the different samples is therefore: reference $>$ dark $>$ photocharged $\mathrm{BiVO}_{4}$.

The difference in screening value between the dark and PC spectra can be explained by the photocharged sample having less electrons in the conduction band than the dark sample in the area measured by XRS, which also explains the pre-edge filling of the dark sample observed in Figure 3. The screening value of 0.2 for $\mathrm{PC}-\mathrm{BiVO}_{4}$ is significantly higher with respect to the value of 0 that was used for the reference sample, showing strong structural changes in $\mathrm{BiVO}_{4}$ as a result of the photocharging treatment. A higher screening value also indicates a shift to a lower Fermi level since less electrons are present in the conduction band. Less electrons for the photocharged sample might seem counterintuitive, but in fact, it is a consequence of improved band bending induced by the heterojunction between $\mathrm{BiVO}_{4}$ and the surface-formed bismuth borate layer. The fact that electrons are driven away from the surface by band bending is responsible for the strong suppression of surface recombination induced by the photocharging treatment. The decrease in Fermi level is also introduced in the simulations. The dark sample also has less electrons compared to the reference sample and a lower Fermi level, but the changes are smaller compared to the photocharged sample. 


\section{DISCUSSION}

The XPS analysis confirmed the formation of a bismuth borate surface layer on $\mathrm{BiVO}_{4}$, upon photocharging. The shifts of the core-level XPS spectra, to lower binding energies upon photocharging, suggested that this borate surface layer induced an upward band bending within $\mathrm{BiVO}_{4}$ (Figure 5a). Similar conclusions could be drawn in the case of the dark samples. After $18 \mathrm{~h}$ under open-circuit conditions in the dark, the dark sample has an $\mathrm{OH}$ coverage that initially improves the photoelectrochemical performance of the sample (Figure S9). This improvement is, however, smaller than the PEC improvement of the PC samples and is not maintained over time because the $\mathrm{OH}$ species are removed from the surface during water oxidation. Both the dark and photocharged samples demonstrated a higher open-circuit potential in the dark than the reference sample (Figure S2), resulting in a Fermi level closer to the conduction band in the bulk, as depicted in Figure 5a. To determine whether the bismuth borate and $\mathrm{OH}$ surface layers had any effect on the bulk structure of $\mathrm{BiVO}_{4}$, both UV-vis and XRS measurements were performed. The UV-vis spectra revealed a change in absorption after photocharging, with the formation of an above band gap absorption feature. This feature was attributed to a defect-related absorption, possibly from the creation of surface oxygen vacancies.

To gain a deeper understanding of the electronic effect of the bismuth borate layer on $\mathrm{BiVO}_{4}$, XRS measurements were performed. These results showed that the hybridized $\mathrm{Bi} 6 \mathrm{p}-\mathrm{O}$ $2 p$ orbital disappeared, which corresponds well to the formation of a bismuth borate surface layer. In addition, the $\mathrm{ab}$ initio simulations of the $\mathrm{O} \mathrm{K}$ edge spectra revealed a decrease in the Fermi level of the $\mathrm{PC}-\mathrm{BiVO}_{4}$. This can be explained by band bending induced by the surface borate layer. Bismuth borate has a larger band gap than $\mathrm{BiVO}_{4}$, and according to the valence band spectra (Figure 1d), its valence band lies closer to the Fermi level than the valence band of $\mathrm{BiVO}_{4}$. This means that the heterojunction of $\mathrm{BiVO}_{4}$ and bismuth borate will create strong band bending and a significant space charge region (SCR) of several tens of nanometers (Figure 5a). ${ }^{42,43}$ The SCR coincides with the region that is probed by XRS. Since the band bending forces electrons toward the back contact, the SCR will contain fewer electrons than in the case of a flat band situation. The monolayer of $\mathrm{OH}$ on the surface of the dark $\mathrm{BiVO}_{4}$ sample has a similar band bending effect that directs the electrons toward the back contact, away from the surface.

The XRS results from this work suggest that there is a severe alteration in the surface electronic structure when $\mathrm{BiVO}_{4}$ comes in contact with an electrolyte, even without illumination. This is also supported by the changes visible in the data obtained from XPS and HR-SEM. The initiator of this surface restructuring could be the surface adsorption of electrolyte anionic species, borate in the case of the PC$\mathrm{BiVO}_{4}$ and hydroxide in the case of dark $\mathrm{BiVO}_{4}$. The heterojunctions formed as a result of this surface adsorption improve the charge separation close to the surface and thus suppress surface recombination. Since other metal oxides $^{13,14,16}$ were also shown to photocharge like $\mathrm{BiVO}_{4}$, these findings could be generalized.

Additionally, this time-dependent surface layer formation shows that the metal oxide/electrolyte interface is very dynamic. Reversibility of the photocharging effect ${ }^{10}$ in the dark could be explained by the desorption of this buffer anion surface layer under dark conditions, which was also shown by Favaro et al. ${ }^{17}$ The dynamic nature of the metal oxide/ electrolyte interface makes it difficult for the photoanode material to be stable for long-term operation. The constant switching between the dark and light conditions, as in a practical case where intermittent sunlight drives the reaction, would imply constant surface restructuring of the photoanode material. Some material from the surface could be lost in each of these restructuring steps, in addition to other corrosion mechanisms occurring at the surface of a photoanode material.

It is important to emphasize that this surface layer formation, in the dark and under illumination, from the electrolyte anionic species has been "ever-present" in metal oxide photoanodes. This work highlights that the choice of electrolyte/buffer solutions needs to be considered when making comparisons of PEC performance of different systems with the same metal oxide semiconductor. The surface layer formed would be different with different electrolytes and hence the resultant heterojunction would change as well. However, the importance or the effect of the electrolyte has been largely unnoted in the PEC research field when analyzing material surfaces after photoelectrochemical measurements. This current study is a strong indicator that the effect of surface adsorption of electrolyte anionic species can have serious implications on PEC measurements and should be given due regard for future works in the field.

\section{CONCLUSIONS}

The photocharging treatment of $\mathrm{BiVO}_{4}$ was studied using a sequence of XPS, UV-vis, and XRS techniques. The XPS studies revealed the formation of a surface borate layer on the $\mathrm{BiVO}_{4}$ surface, upon illumination in open-circuit conditions. The borate anion from the electrolyte covalently bonded with the bismuth ions in the $\mathrm{BiVO}_{4}$, resulting in a $\mathrm{BiVO}_{4} /$ bismuth borate heterojunction near the surface. This heterojunction resulted in improved band bending near the surface, improving the charge separation and suppressing the surface recombination of charge carriers. The improved band bending, as a result of the heterojunction, explains the enhancement in the PEC performance of photocharged $\mathrm{BiVO}_{4}$. In a similar manner, a $\mathrm{BiVO}_{4}$ sample kept under open-circuit conditions in the dark formed an $\mathrm{OH}$ layer at the surface, leading to a relatively smaller degree of band bending and a short-lived PEC performance enhancement.

The UV-vis studies showed the formation of a new absorption feature, outside the band gap of $\mathrm{BiVO}_{4}$, upon photocharging. This feature was attributed to a defect-related absorption, resulting from the surface restructuring during photocharging. This surface restructuring during photocharging was confirmed using HR-SEM images. A combination of XRS studies and $a b$ initio simulations on the oxygen $\mathrm{K}$ edge spectra revealed a decrease in the electron occupancy in the space charge region of the dark and ${\mathrm{PC}-\mathrm{BiVO}_{4}}$ samples, confirming the improvement in band bending after the dark and photocharging treatment. Additionally, the XPS, UV-vis, and XRS measurements indicated a decrease in the oxidation state of the surface vanadium species due to the anion adsorption. Further experiments are required to quantify this change.

A strong agreement was found between the XRS spectra obtained in this work and soft X-ray XANES spectra from others, indicating that XRS is a very powerful tool to study 
semiconductors in (near) in situ conditions with a larger probing depth than allowed by soft X-ray techniques. The dynamic nature of the metal oxide/electrolyte interface will have strong implications on the long-term stability of metal oxide photoanodes. The effect of surface adsorption of electrolyte anionic species on the surface structure of metal oxide photoelectrodes is often overlooked within the PEC research field. Our results show that this time-dependent surface adsorption of electrolyte anionic species on the metal oxide photoanode surface plays an important role in defining the electronic and catalytic properties of a photoanode. Anionic adsorption was shown to form a heterojunction at the surface, which improved charge separation and suppressed surface recombination. The effect of the electrolyte on the metal oxide photoanode surface and the time scale of the surface adsorption of these anions cannot be ignored, and hence should be taken into consideration while performing analyses and gaining mechanistic understanding of reactions on metal oxide photoanode surfaces.

\section{ASSOCIATED CONTENT}

\section{S Supporting Information}

The Supporting Information is available free of charge on the ACS Publications website at DOI: 10.1021/acs.chemmater.9b02121.

Additional XPS spectra and UV-vis spectra, HR-SEM image, XRD pattern, XRS simulations, and FDMNES input file; PEC performance data; and band gap calculation (PDF)

\section{AUTHOR INFORMATION}

\section{Corresponding Authors}

*E-mail: alessandro.longo@esrf.fr. (A.L.).

*E-mail: w.smith@tudelft.nl. (W.S.).

\section{ORCID}

Nienke J. Firet: 0000-0002-2808-4598

Marijn A. Blommaert: 0000-0003-1568-0961

Christoph J. Sahle: 0000-0001-8645-3163

Wilson A. Smith: 0000-0001-7757-5281

\section{Author Contributions}

${ }^{\perp}$ N.J.F. and A.V. contributed equally to this work. The manuscript was written through contributions of all authors. All authors have given approval to the final version of the manuscript.

\section{Notes}

The authors declare no competing financial interest.

\section{ACKNOWLEDGMENTS}

This work was financed by the Netherlands Organisation for Scientific Research (NWO, project number FOM-13CO19). The authors acknowledge ESRF for funding of the synchrotron experiments and C. Henriquet for technical support at ID20. They thank Marcus Fehse for fruitful discussions, Joost Middelkoop for cell design, and Nathan Nesbitt for taking HR-SEM images.

\section{REFERENCES}

(1) Alexander, B. D.; Kulesza, P. J.; Rutkowska, I.; Solarska, R.; Augustynski, J. Metal Oxide Photoanodes for Solar Hydrogen Production. J. Mater. Chem. 2008, 18, 2298-2303.
(2) Han, H. S.; Shin, S.; Kim, D. H.; Park, I. J.; Kim, J. S.; Huang, P. S.; Lee, J. K.; Cho, I. S.; Zheng, X. Boosting the Solar Water Oxidation Performance of a BiVO4 Photoanode by Crystallographic Orientation Control. Energy Environ. Sci. 2018, 11, 1299-1306.

(3) Abdi, F. F.; Han, L.; Smets, A. H. M.; Zeman, M.; Dam, B.; Van De Krol, R. Efficient Solar Water Splitting by Enhanced Charge Separation in a Bismuth Vanadate-Silicon Tandem Photoelectrode. Nat. Commun. 2013, 4, No. 2195.

(4) Sharp, I. D.; Cooper, J. K.; Toma, F. M.; Buonsanti, R. Bismuth Vanadate as a Platform for Accelerating Discovery and Development of Complex Transition-Metal Oxide Photoanodes. ACS Energy Lett. 2017, 2, 139-150.

(5) Pilli, S. K.; Furtak, T. E.; Brown, L. D.; Deutsch, T. G.; Turner, J. A.; Herring, A. M. Cobalt-Phosphate (Co-Pi) Catalyst Modified MoDoped BiVO4 Photoelectrodes for Solar Water Oxidation. Energy Environ. Sci. 2011, 4, 5028-5034.

(6) Zhong, D. K.; Choi, S.; Gamelin, D. R. Near-Complete Suppression of Surface Recombination in Solar Photoelectrolysis by "Co-Pi" Catalyst-Modified W:BiVO4. J. Am. Chem. Soc. 2011, 133, 18370-18377.

(7) Lee, D.; Kvit, A.; Choi, K. S. Enabling Solar Water Oxidation by BiVO4 Photoanodes in Basic Media. Chem. Mater. 2018, 30, 47044712.

(8) Zachäus, C.; Abdi, F. F.; Peter, L. M.; Van De Krol, R. Photocurrent of BiVO4 Is Limited by Surface Recombination, Not Surface Catalysis. Chem. Sci. 2017, 8, 3712-3719.

(9) Trotochaud, L.; Mills, T. J.; Boettcher, S. W. An Optocatalytic Model for Semiconductor-Catalyst Water-Splitting Photoelectrodes Based on in Situ Optical Measurements on Operational Catalysts. J. Phys. Chem. Lett. 2013, 4, 931-935.

(10) Trześniewski, B. J.; Smith, W. A. Photocharged BiVO4 Photoanodes for Improved Solar Water Splitting. J. Mater. Chem. A 2016, 4, 2919-2926.

(11) Trześniewski, B. J.; Digdaya, I. A.; Nagaki, T.; Ravishankar, S.; Herraiz-Cardona, I.; Vermaas, D. A.; Longo, A.; Gimenez, S.; Smith, W. A. Near-Complete Suppression of Surface Losses and Total Internal Quantum Efficiency in BiVO4 Photoanodes. Energy Environ. Sci. 2017, 10, 1517-1529.

(12) Liu, E. Y.; Thorne, J. E.; He, Y.; Wang, D. Understanding Photocharging Effects on Bismuth Vanadate. ACS Appl. Mater. Interfaces 2017, 9, 22083-22087.

(13) Breuhaus-Alvarez, A. G.; Dimeglio, J. L.; Cooper, J. J.; Lhermitte, C. R.; Bartlett, B. M. Kinetics and Faradaic Efficiency of Oxygen Evolution on Reduced HxWO3 Photoelectrodes. J. Phys. Chem. C 2019, 123, 1142-1150.

(14) Venugopal, A.; Smith, W. A. Light Induced Formation of a Surface Hetero-Junction in Photocharged CuWO4 Photoanodes. Faraday Discuss. 2019, 215, 175-191.

(15) Xie, J.; Yang, P.; Liang, X.; Xiong, J. Self-Improvement of Ti:Fe2O3 Photoanodes: Photoelectrocatalysis Improvement after Long-Term Stability Testing in Alkaline Electrolyte. ACS Appl. Energy Mater. 2018, 1, 2769-2775.

(16) Deng, J.; Lv, X.; Zhong, J. Photocharged Fe2TiO5/Fe2O3 Photoanode for Enhanced Photoelectrochemical Water Oxidation. J. Phys. Chem. C 2018, 122, 29268-29273.

(17) Favaro, M.; Abdi, F. F.; Lamers, M.; Crumlin, E. J.; Liu, Z.; van de Krol, R.; Starr, D. E. Light-Induced Surface Reactions at the Bismuth Vanadate/Potassium Phosphate Interface. J. Phys. Chem. B 2018, 122, 801-809.

(18) Abdi, F. F.; Firet, N.; van de Krol, R. Efficient BiVO4 Thin Film Photoanodes Modified with Cobalt Phosphate Catalyst and WDoping. ChemCatChem 2013, 5, 490-496.

(19) Huotari, S.; Sahle, C. J.; Henriquet, C.; Al-Zein, A.; Martel, K.; Simonelli, L.; Verbeni, R.; Gonzalez, H.; Lagier, M. C.; Ponchut, C.; et al. A Large-Solid-Angle X-Ray Raman Scattering Spectrometer at ID20 of the European Synchrotron Radiation Facility. J. Synchrotron Radiat. 2017, 24, 521-530.

(20) Singh, S. P.; Karmakar, B. Bismuth Oxide Doped Glasses for Optical and Photonic Applications. In Bismuth: Characteristics, 
Production and Applications; Materials Science and Technologies: NY, 2012; Chapter 9.

(21) Sahle, C. J.; Mirone, A.; Niskanen, J.; Inkinen, J.; Krisch, M.; Huotari, S. Planning, Performing and Analyzing X-Ray Raman Scattering Experiments. J. Synchrotron Radiat. 2015, 22, 400-409.

(22) Joly, Y. X-Ray Absorption near-Edge Structure Calculations beyond the Muffin-Tin Approximation. Phys. Rev. B 2001, 63, No. 125120

(23) Joly, Y.; Cavallari, C.; Guda, S. A.; Sahle, C. J. Full-Potential Simulation of X-Ray Raman Scattering Spectroscopy. J. Chem. Theory Comput. 2017, 13, 2172-2177.

(24) Retegan, M. Mretegan/Crispy, v0.7.1.; Zenobo, 2018.

(25) Oprea, B.; Radu, T.; Simon, S. XPS Investigation of Atomic Environment Changes on Surface of B2O3-Bi2O3 Glasses. J. NonCryst. Solids 2013, 379, 35-39.

(26) Bajaj, A.; Khanna, A.; Chen, B.; Longstaffe, J. G.; Zwanziger, U. W.; Zwanziger, J. W.; Gómez, Y.; González, F. Structural Investigation of Bismuth Borate Glasses and Crystalline Phases. J. Non-Cryst. Solids 2009, 355, 45-53.

(27) Hermans, Y.; Murcia-López, S.; Klein, A.; Morante, J. R.; van de Krol, R.; Andreu, T.; TOUPANCE, T.; Jaegermann, W. Analysis of the Interfacial Characteristics of BiVO4/Metal Oxide Heterostructures and Its Implication on Their Junction Properties. Phys. Chem. Chem. Phys. 2019, 21, 5086-5096.

(28) Feldman, L. C.; Boatner, L. A.; López, R.; Rini, M.; Haynes, T. E.; Cavalleri, A.; Haglund, R. F.; Schoenlein, R. W. Photoinduced Phase Transition in VO2 Nanocrystals: Ultrafast Control of SurfacePlasmon Resonance. Opt. Lett. 2005, 30, 558-560.

(29) Schubert, W. K.; Wolf, E. L. Electron-Energy-Loss Spectra of Vanadium, Niobium, Molybdenum, and Tantalum. Phys. Rev. B 1979, $20,1855-1862$

(30) Toudert, J.; Serna, R.; Jiménez De Castro, M. Exploring the Optical Potential of Nano-Bismuth: Tunable Surface Plasmon Resonances in the near Ultraviolet-to-near Infrared Range. J. Phys. Chem. C 2012, 116, 20530-20539.

(31) Wang, L.; Tsang, C. S.; Liu, W.; Zhang, X.; Zhang, K.; Ha, E.; Kwok, W. M.; Park, J. H.; Suk Lee, L. Y.; Wong, K. Y. Disordered Layers on WO 3 Nanoparticles Enable Photochemical Generation of Hydrogen from Water. J. Mater. Chem. A 2019, 7, 221-227.

(32) Wang, S.; Chen, P.; Yun, J. H.; Hu, Y.; Wang, L. An Electrochemically Treated BiVO4 Photoanode for Efficient Photoelectrochemical Water Splitting. Angew. Chem., Int. Ed. 2017, 56, $8500-8504$.

(33) Jovic, V.; Laverock, J.; Rettie, A. J. E.; Zhou, J.-S.; Mullins, C. B.; Singh, V. R.; Lamoureux, B.; Wilson, D.; Su, T.-Y.; Jovic, B.; et al. Soft X-Ray Spectroscopic Studies of the Electronic Structure of M:BiVO 4 (M = Mo, W) Single Crystals. J. Mater. Chem. A 2015, 3, 23743-23753.

(34) Cooper, J. K.; Gul, S.; Toma, F. M.; Chen, L.; Glans, P. A.; Guo, J.; Ager, J. W.; Yano, J.; Sharp, I. D. Electronic Structure of Monoclinic BiVO4. Chem. Mater. 2014, 26, 5365-5373.

(35) Jovic, V.; Rettie, A. J. E.; Singh, V. R.; Zhou, J.; Lamoureux, B.; Buddie Mullins, C.; Bluhm, H.; Laverock, J.; Smith, K. E. A Soft XRay Spectroscopic Perspective of Electron Localization and Transport in Tungsten Doped Bismuth Vanadate Single Crystals. Phys. Chem. Chem. Phys. 2016, 18, 31958-31965.

(36) Zhao, Z.; Li, Z.; Zou, Z. Electronic Structure and Optical Properties of Monoclinic Clinobisvanite BiVO4. Phys. Chem. Chem. Phys. 2011, 13, 4746-4753.

(37) Nie, K.; Kashtanov, S.; Wei, Y.; Liu, Y. S.; Zhang, H.; Kapilashrami, M.; Ye, Y.; Glans, P. A.; Zhong, J.; Vayssieres, L.; et al. Atomic-Scale Understanding of the Electronic Structure-Crystal Facets Synergy of Nanopyramidal CoPi/BiVO4 Hybrid Photocatalyst for Efficient Solar Water Oxidation. Nano Energy 2018, 53, 483-491.

(38) Lin, X. W.; Wang, Y. Y.; Dravid, V. P.; Michalakos, P. M.; Kung, M. C. Valence States and Hybridization in Vanadium Oxide Systems Investigated by Transmission Electron-Energy-Loss Spectroscopy. Phys. Rev. B 1993, 47, 3477-3481.
(39) Rossell, M. D.; Agrawal, P.; Borgschulte, A.; Hébert, C.; Passerone, D.; Erni, R. Direct Evidence of Surface Reduction in Monoclinic BiVO 4. Chem. Mater. 2015, 27, 3593-3600.

(40) Tokunaga, S.; Kato, H.; Kudo, A. Selective Preparation of Monoclinic and Tetragonal BiVO4 with Scheelite Structure and Their Photocatalytic Properties. Chem. Mater. 2001, 13, 4624-4628.

(41) Joly, Y.; Cabaret, D.; Renevier, H.; Natoli, C. R. Electron Population Analysis by Full-Potential X-Ray Absorption Simulations. Phys. Rev. Lett. 1999, 82, 2398-2401.

(42) Peter, L. M.; Gurudayal; Wong, L. H.; Abdi, F. F. Understanding the Role of Nanostructuring in Photoelectrode Performance for Light-Driven Water Splitting. J. Electroanal. Chem. 2018, 819, 447-458.

(43) van der Krol, R.; Grätzel, M. Photoelectrochemical Hydrogen Production, 1st ed.; Springer: NY, 2012.

\section{NOTE ADDED AFTER ASAP PUBLICATION}

Due to a production error, this paper was published on the Web on August 14, 2019, with the first co-author name misspelled. The corrected version was reposted on August 16, 2019. 